more, which has become possible in the past few years, may produce FETs with a different gating mechanism and perhaps dramatically better characteristics than those made with more common $67 \%$ to
$75 \%$ semiconducting mixes. Further characterization of field-modulated transport between metallic and semiconducting tubes, or through semiconducting tubes crossed by metallic tubes, will be important in determining whether the predominantly semiconducting networks really are better."

STEVEN TROHALAKI

\section{Polyferrocenylsilanes Enable NIL-Fabricated Magneto-Ceramic Composite Nanorod Arrays}

Driven by the need for faster and cheaper memory, the computer industry has an insatiable appetite for improved magnetic data storage. Recently, K. Liu, S. Fournier-Bidoz, and G.A. Ozin of the University of Toronto and I. Manners of the University of Bristol have developed a magnetic material that has potential for next-generation data storage applications. The material combines the use of high ceramic yield polyferrocenylsilanes (PFSs) and nanoimprint lithography (NIL) to make ordered arrays of magnetic pillars. The researchers' technique allows them to select the dimensions of the PFS material using well-defined anodic aluminum oxide (AAO) templates.

In a recent issue of Chemistry of Materials (DOI: 10.1021/cm900164b; Web publication: April 6), the researchers describe their use of aluminum-backed AAO templates to imprint patterns into PFS at elevated temperatures $\left(150^{\circ} \mathrm{C}\right)$. Once raised above its glass transition temperature, the polymer melt was able to infiltrate the AAO template. The research team found that the extent of template filling was dependent on the temperature ramping rate. Under fast ramping conditions $\left(15^{\circ} \mathrm{C} / \mathrm{min}\right)$, the polymer partially filled the template but was able to completely fill the channels under slower ramping conditions $\left(2^{\circ} \mathrm{C} / \mathrm{min}\right)$.

In order to form magnetic patterned ceramic features, the group melt-filled the AAO with polymer under $\mathrm{N}_{2} / \mathrm{H}_{2}$ $(95 \% / 5 \%)$ to avoid the formation of iron oxides. After cooling the template and removing the aluminum backing, the researchers heated the filled template to between $500^{\circ} \mathrm{C}$ and $800^{\circ} \mathrm{C}$. They removed the AAO template by dissolution in $\mathrm{NaOH}$ to reveal the patterned magnetic ceramic material. Of the three PFSs studied, only the polymer containing the acetylenic substituent gave a high enough ceramic yield (85\%) to provide shape retention.

At elevated temperatures, pyrolysis of PFS forms $\alpha$-Fe nanoparticles that have a theoretical domain size of $14 \mathrm{~nm}$. The researchers found that they were able to tune the magnetic properties of the particles by varying the diameter of the AAO template. When pyrolyzed in templates with $55 \mathrm{~nm}$ pores, the resulting particles had an average size of $8.2 \mathrm{~nm} \pm 2.4 \mathrm{~nm}$ and exhibited superparamagnetic behavior; whereas, polymer pyrolyzed in templates with $95 \mathrm{~nm}$ pores resulted in particles with average diameters of $20.9 \mathrm{~nm} \pm 4.9 \mathrm{~nm}$ and exhibited ferromagnetic behavior. The group hypothesizes that the "growth of the nanoparticles was confined by the diameter of the nanochannels."

KeVIN P. HeRLIHY

\section{Confocal Annular Aperture Microscopy and NAIL Allow High Lateral Resolution in Backside Imaging of Integrated Circuits}

Optical methods for detection of defects in silicon integrated circuits (IC) typically rely on light at wavelengths greater than $1 \mu \mathrm{m}$, where silicon is relatively transparent, which limits the lateral resolution to $\sim 1 \mu \mathrm{m}$. F.H. Koklu, B.B. Goldberg, and M.S. Ünlü from Boston University, and S.B. Ippolito from the IBM Semiconductor Research \& Development Center in Hopewell Junction, New York, achieved a better imaging resolution by combining a confocal laser scanning microscope with a silicon numerical aperture increasing lens (NAIL). A silicon NAIL placed on the backside of a silicon substrate effectively transforms it into an integrated solid immersion lens with increased numerical aperture (NA) of a factor of the square of the refractive index to a maximum of 3.5 , the refractive index of silicon. Confocal laser scanning microscopes yield a lateral spatial resolution determined by the spot size of the laser beam. By combining the two techniques in conjunction with angular spectrum engineering, the researchers achieved a lateral spatial resolution of $145 \mathrm{~nm}$ at $\lambda_{0}=$ $1.3 \mu \mathrm{m}$, which represents a resolution of $\sim \lambda_{0} / 9$, as they reported in the April 15 issue of Optics Letters (DOI: 10.1364/ OL.34.001261; p. 1261).

The confocal microscopy setup the researchers developed is a single-path, reflection-mode fiber-optical scanning microscope that uses a single mode fibercoupled laser diode emitting at $1.3 \mu \mathrm{m}$ and a $2 \times 2$ optical coupler. The researchers coupled the light in and out the single mode fiber with a collimating objective with matching NA and illuminated the sample and the NAIL using a second objective with $\mathrm{NA}=0.26$, the same objective that collected the reflected signal. The NAIL consisted of an undoped silicon hemisphere with radius of 1.61 $\mathrm{mm}$. The images were generated by scanning the sample with the NAIL using a piezo translation stage. The researchers controlled the polarization direction of the incoming light with a half-wave plate located before the imaging objective. They modified the angular spectrum by blocking the center of the optical path in front of the imaging objective in such a way that they formed an annular aperture of variable inner radius. With this setup, they scanned an IC fabricated in a $0.35 \mu \mathrm{m}$ process with 4 metal layers and 2 polysilicon layers deposited on a silicon substrate. The silicon substrate was thinned to a thickness of $458 \mu \mathrm{m} \pm 2 \mu \mathrm{m}$ in order to optimize the resolution and imaging quality of the combined imaging approach. The structures imaged were passive calibration structures embedded into the first polysilicon layer.

The researchers were able to demonstrate that by tailoring the angular spectrum with an annular aperture while using linearly polarized illumination it was possible to significantly improve the spatial resolution in one direction. The research team achieved a record lateral spatial resolution of $145 \mathrm{~nm}$ in the direction perpendicular to the polarization direction of the incoming light for onephoton excitation schemes by engineering the pupil function. The researchers said that together with its contribution to longitudinal localization, angular spectrum tailoring proved to be a powerful and simple technique to improve the optical inspection of ICs.

JOAN J. CARVAJAL 